\title{
Non-Viral Engineering of CAR-NK and CAR-T cells using the Tc Buster Transposon System ${ }^{\text {TM }}$
}

Emily J. Pomeroy ${ }^{1,2,3,4}$, Walker S. Lahr ${ }^{1,2,3,4}$, Jae Woong Chang ${ }^{1,2,3,4}$, Joshua Krueger ${ }^{1,2,3,4}$, Bryce J. Wick ${ }^{1,2,3,4}$, Nicholas J. Slipek ${ }^{1,2,3,4}$, Joseph G. Skeate ${ }^{1,2,3,4}$, Beau R. Webber ${ }^{1,2,3,4}$, Branden S. Moriarity ${ }^{1,2,3,4}$

${ }^{1}$ Department of Pediatrics, University of Minnesota

${ }^{2}$ Masonic Cancer Center, University of Minnesota

${ }^{3}$ Center for Genome Engineering, University of Minnesota

${ }^{4}$ Stem Cell Institute, University of Minnesota

\begin{abstract}
Cancer immunotherapy using $\mathrm{T}$ cells and NK cells modified with viral vectors to express a chimeric antigen receptor (CAR) has shown remarkable efficacy in treating hematological malignancies in clinical trials. However, viral vectors are limited in their cargo size capacity, and large-scale manufacturing for clinical use remains complex and cost prohibitive. As an alternative, CAR delivery via DNA transposon engineering is a superior and cost-effective production method. Engineering via transposition is accomplished using a two-component system: a plasmid containing a gene expression cassette flanked by transposon inverted terminal repeats (ITRs) paired with a transposase enzyme that binds to the ITRs, excises the transposon from the plasmid, and stably integrates the transposon into the genome.
\end{abstract}

Here, we used the newly developed hyperactive Tc Buster (Bio-Techne) transposon system to deliver a transposon containing a multicistronic expression cassette (CD19-CAR, mutant DHFR, and EGFP) to primary human peripheral blood (PB) NK cells and T cells. We optimized methods to avoid DNA toxicity and maximize efficiency. 
Our cargo contained a mutant dihydrofolate reductase (DHFR) which allowed us to enrich for stable transposon integration using methotrexate (MTX) selection. We then tested CAR-NK and CAR-T cells in functional assays against CD19-expressing Raji cells. CAR-expressing $\mathrm{NK}$ and $\mathrm{T}$ cells produced significantly more cytokines than CAR-negative controls and efficiently killed target cells. We recognize that cryopreservation manufactured CAR-expressing cells will be necessary for clinical translation. We observed reduced cytotoxicity of CAR-NK cells immediately after thaw, but increasing the NK dose overcame this loss of function.

Our work provides a platform for robust delivery of multicistronic, large cargo via transposition to primary human NK and $\mathrm{T}$ cells. We demonstrate that CAR-expressing cells can be enriched using MTX selection, while maintaining high viability and function. This non-viral approach represents a versatile, safe, and cost-effective option for the manufacture of CAR-NK and CAR-T cells compared to viral delivery.

\section{Introduction}

DNA transposons are natural DNA transfer vehicles that can be used for DNA delivery into human cells. In nature, they exist as well-defined elements in which the transposase gene is flanked by inverted terminal repeats (ITRs) that encode transposase binding sites. They can be used as a tool for stable genomic insertion by flanking an expression cassette with ITRs and co-delivering the transposase enzyme via an expression plasmid, mRNA, or protein ${ }^{1}$. 
Several DNA transposons have been used in such a manner in mammalian cells.

In the 1990s, the Sleeping Beauty (SB) transposon system was molecularly reconstructed by eliminating inactivating mutations found in members of the Tc1/mariner family of transposons isolated from fish ${ }^{2}$. This reactivated transposon system has since been used for stable gene transfer and insertional mutagenesis in many vertebrate cell types, including human cells. Subsequently, the piggyBac $(P B)$ and Tol2 transposable elements were isolated from insects and fish, respectively, and have been optimized for enhanced activity in mammalian cells ${ }^{3,4} . S B, P B$, and Tol2 can all be used as efficient non-viral tools for stable gene delivery, and each of these has been used for gene delivery in primary human lymphocytes ${ }^{5}$. The hAT superfamily of transposons (including Tol2) are found in diverse species ${ }^{6}$. A novel representative of this family is Tc Buster, originally isolated from the red flour beetle ${ }^{7}$. Tc Buster has been shown to be active in human cell lines and has a comparable transposition efficiency to $S B$ and $P B^{6,8}$. A hyperactive mutant of $T c$ Buster was recently developed (Bio-Techne, Minneapolis, MN) for the non-viral manufacture of cellular therapies.

Transposons have many meaningful advantages as an alternative to viral vectors for stable gene transfer. Several clinical gene therapy products have been developed using CD34+ hematopoietic stem cells or $\mathrm{T}$ cells genetically modified using recombinant viruses; namely $\gamma$-retroviruses, and lentiviruses ${ }^{\text {9-19. }}$. These delivery methods carry the risk of insertional mutagenesis via activation of proto-oncogenes or inactivation of tumor suppressor genes ${ }^{20-22}$. In addition, large-scale manufacturing of these viral vectors for 3 
clinical use is cost-prohibitive, inconsistent, and impedes progression through clinical trials; particularly for academic institutions and emerging biotech companies. The use of transposon systems has been pursued as an alternative to viral vectors due to rapid, reproducible, and cost-effective production and a favorable safety profile ${ }^{10,20,23}$. However, any vector that integrates into chromosomes poses the risk of insertional mutagenesis. A comparative study of the target site integration properties of $S B$ and $P B$ transposons as well as gammaretroviral and lentiviral systems in primary human CD4+ T cells ranked their safety profiles based on multiple criteria, including distance from the 5'-end of any gene and distance from any cancer-related gene. This analysis established $S B$ as having the most favorable integration profile, followed by $P B$, suggesting that engineering via transposition is a safer alternative to engineering with viral vectors ${ }^{24}$. Further, genome wide analysis comparing integrations of Tc Buster to piggyBac and Sleeping Beauty revealed a unique but comparable integration pattern for Tc Buster ${ }^{6}$.

The use of transposon systems for gene delivery in human lymphocytes has been most widely studied as a method for generating human $\mathrm{T}$ cells engineered to express $\mathrm{CARs}^{25,26}$. Clinically, the $S B$ system has been used to introduce CD19-specific CARs to patient- and donor-derived $\mathrm{T}$ cells $\mathrm{s}^{27,28}$. Many preclinical studies and clinical trials thus far have introduced $S B$ transposase and CAR by electroporation of bulk peripheral blood mononuclear cells (PBMCs) ${ }^{\mathbf{2 9}-33}$. CAR-expressing $\mathrm{T}$ cells were subsequently expanded over several weeks in culture using feeder cells engineered to express the target antigen and co-stimulatory molecules ${ }^{27}$. Efforts are now being made to shorten the culture time 4 
before patient infusion (NCT04102436, NCT04289220). One such trial is underway in which PBMCs are transferred into the patient within 2 days after electroporation with $S B$ transposase, CD19-CAR, and membrane-bound IL15 (NCT03579888). Signaling through the CAR and mbIL15 gives genetically-modified $\mathrm{T}$ cells a selective advantage after transplant, fostering their outgrowth ${ }^{34}$.

Another approach for shortening culture time ex vivo is to electroporate $\mathrm{T}$ cells directly, rather than as bulk PBMCs from which T cells need to be selected. This has been challenging as the delivery of transposons via plasmids leads to DNA toxicity in T cells due to a type I interferon (IFN) response ${ }^{35,36}$. Thus, efforts have been made to minimize the amount of DNA introduced to the $\mathrm{T}$ cell. Minicircle vectors are DNA plasmid delivery vehicles that do not carry a bacterial origin of replication or bacterial resistance genes, reducing the size of the vector to only that of the expression cassette ${ }^{37}$ This approach has been used to achieve stable expression of transgenes in primary human $\mathrm{T}$ cells with efficiencies over 50 percent ${ }^{35,38,39}$. However, minicircle vectors rely on a complex and inefficient recombination-based removal of the plasmid bacterial regions prior to plasmid purification, often leading to low plasmid yield, bacterial genomic DNA contamination, and overall inconsistency between productions. More recently, Nanoplasmids (Nature Technology) have been developed that contain a small ( $<500 \mathrm{bp})$ backbone that encodes the origin of replication from the Rep/iteron plasmid $\mathrm{R} 6 \mathrm{~K}^{\mathbf{4 0}}$ and the RNA-OUT (antisense RNA) selectable marker ${ }^{41}$. This minimal backbone reduces 
DNA toxicity and thus nanoplasmids can be made with high yield and without complex purification methods $\mathrm{s}^{\mathbf{4 1 , 4 2}}$.

Thus far, the use of transposons for NK cells has been mostly applied to the NK-92 cell line ${ }^{43}$. Recently, the $S B$ transposon system has been used to deliver a CAR to cytokine-induced killer cells for targeting CD33 on chemoresistance acute myeloid leukemia (AML) in patient-derived xenografts ${ }^{\mathbf{1}, \mathbf{4 4}}$. However, lessons can be learned from $\mathrm{T}$ cells on the use of transposons for CAR delivery to primary NK cells. The initial approach of electroporating PBMCs with transposon-based CARs suggests that NK cells could be selectively outgrown instead of $\mathrm{T}$ cells $\mathrm{s}^{\mathbf{2 9}, \mathbf{3 0}}$. Indeed, some reports have shown outgrowth of NK cells reaching 50\% of the PBMC population after co-culture with feeder cells ${ }^{\mathbf{3 0}}$. Thus, this approach could be optimized for selection of CAR-expressing NK cells, or delivery of a mixed population of CAR-T and -NK cells might be advantageous as NK cells have been shown to produce inflammatory cytokines to help shape the adaptive immune response ${ }^{45}$.

Alternatively, the use of minicircle or nanoplasmid vectors to deliver transposons directly to purified NK cells is an attractive option. NK cells share many properties with T cells, and delivery of DNA to NK cells has been shown to induce similar toxicity ${ }^{\mathbf{4}}$. Thus, reducing the amount of DNA delivery by using mRNA-encoded transposase in combination with a minicircle- or nanoplasmid-encoded transposon may be ideal.

The use of transposons for engineering NK cells is not limited to the delivery of CARs. Other modifications have been proposed to enhance aspects of NK cell activity 6 
including persistence, migration, and cytotoxicity. This includes the introduction of self-stimulating cytokine receptors ${ }^{47}$, strong activating receptors, or dominant negative versions of $\mathrm{NK}$ cell inhibitory receptors ${ }^{\mathbf{4 8}}$. Such modifications could be used in combination with CAR delivery to create an NK cell expertly equipped to kill a broad range of tumor types. Transposons provide a non-viral strategy to introduce all of these transgenes in one vector that could be scaled up for clinical use. Here we developed such a method for clinically scalable production of CAR-NK and CAR-T cells using the $T c$ Buster Transposon System. We achieve manufacturing of enriched (>99\%+; 46\%+ pre-MTX selection) CAR-expressing cells in a matter of 3 weeks and show functional efficacy of CD19 targeting CAR-NK and CAR-T cells. Our work provides a versatile, cost-effective, and clinically scalable platform for the manufacture of engineered NK and T cells.

\section{Results}

\section{Optimized Engineering of CAR-NK Cells using Hyperactive Tc Buster}

In an effort develop a robust platform for the manufacture and enrichment of CAR-expressing NK and T cells, we used the newly developed hyperactive Tc Buster ${ }^{\mathrm{TM}}$ (TcB-M, Bio-Techne) system to deliver a nanoplasmid containing a CD19-CAR-DHFR-EGFP expression cassette (3.7 kb transposon, Figure 1A) to primary human peripheral blood (PB) NK cells. 
We previously developed a method for efficient RNA delivery of CRISPR-Cas9 to primary human peripheral blood (PB) NK cells ${ }^{49}$. We used this as a starting point for optimization of transposition. Briefly, we stimulated NK cells by co-culture with membrane bound IL21 (mbIL21)- and 41BBL-expressing K562 feeder cells for 7 days before electroporating them with mRNA encoding Cas 9 and chemically modified sgRNA using the Neon Transfection System ${ }^{49}$. We initially followed this same process to deliver the nanoplasmid transposon and mRNA encoding Tc Buster transposase. We observed low delivery efficiency as measured by GFP expression $(2.07 \pm 0.37 \%)$ and poor recovery $(12.00 \pm 0.58 \%)$ of electroporated cells (Supplemental Figure 1A and 1B).

Suspecting that cytosolic DNA sensors may be upregulated in NK cells during the activation process leading to poor delivery efficiency and cell recovery ${ }^{\mathbf{5 0}}$, we surmised that delivering DNA earlier in the activation process may precede the upregulation of DNA sensors. Thus, we tested delivery of the transposon with Tc Buster (TcB) or the hyperactive Tc Buster mutant transposase (TcB-M) on day 2, 3, or 4 of feeder cell-mediated activation (Figure 1B). In line with our hypothesis, we observed higher transposition efficiency at earlier time points, with the highest efficiency on day 4 of activation $(11.85 \pm 1.26 \%$ for $\mathrm{TcB}$ and $24.05 \pm 1.36 \%$ for $\mathrm{TcB}-\mathrm{M})$. Importantly, the majority of feeder cells in the population are eliminated by day 4 of the activation process (Supplemental Figure 2). 
Activation of NK cells is known to lead to the upregulation of ribonucleases ${ }^{\mathbf{5 1}}$, and as we deliver the transposase as mRNA, we tested the treatment of NK cells with a ribonuclease inhibitor (RNase $\mathrm{A}$, RNase $\mathrm{B}$, and RNase $\mathrm{T} 2$ ) for 5 minutes prior to electroporation with transposase mRNA (Figure $1 \mathrm{C}-1 \mathrm{E}$ ). We found that the addition of the RNase inhibitor enhanced transposition efficiency, and again observed the highest transposition efficiency when electroporation was performed on day 4 of NK cell activation (Figure 1C, $13.23 \pm 1.89 \%$ for $\mathrm{TcB}$ and $49.63 \pm 4.64 \%$ for TcB-M). We re-expanded the NK cells 48-hours after electroporation for one week and compared viability and fold-expansion for each electroporation time-point. While differences in cell viability were minimal (Figure 1D), NK cells electroporated on day 4 of activation had significantly higher fold-expansion than those electroporated on day 2 or day 3 (Figure 1E). Thus, optimal stable transposition in NK cells was achieved by electroporation on day 4 of expansion and pre-treatment with RNase inhibitor before electroporation.

\section{Enrichment of CAR-NK Cells using Methotrexate}

The antifolate methotrexate (MTX) inhibits wild-type dihydrofolate reductase (DHFR), which is essential for cell growth and proliferation ${ }^{52,53}$. Our cargo contained a MTX-resistant DHFR mutant (L22F, F31S) ${ }^{\mathbf{5 4}}$, allowing us to select and expand NK cells that had undergone stable transposon integration. This approach is being used clinically to enrich for CAR-expressing T cells (NCT04483778). We incorporated this selection 
step into our production process during an additional round of feeder cell-mediated expansion (Figure 2A). Three doses of MTX were evaluated to determine the minimal dose needed to kill control cells and enrich engineered cells while maintaining high cell viability and recovery (Figure $2 \mathrm{~B}$ and $2 \mathrm{C}$ ). An optimal dose of $250 \mathrm{nM}$ MTX completely killed control cells (Figure 2B) and enriched cells engineered with either TcB or TcB-M to $>99 \%$ GFP+ (Figure 2C). CD19-CAR expression was confirmed by staining cells with Atto 647N-labeled recombinant human CD19 (Supplemental Figure 3A). Our optimized protocol achieves manufacturing in $\sim 20$ days and results in $99.2 \%( \pm 0.5 \%) \mathrm{CAR}+\mathrm{NK}$ cells expanded 1380-fold ( \pm 104.4) from electroporation input (Figure 2D).

\section{Functional Validation of CAR-NK Cells}

We evaluated NK cells engineered with TcB-M and selected to $>99 \% \mathrm{CAR}+$ with MTX for testing in functional assays against the CD19-expressing Raji Burkitt's lymphoma cell line. NK cells electroporated with the transposon nanoplasmid alone (without transposase) and expanded without MTX selection served as CAR-negative controls. CAR-negative or CAR-positive NK cells were co-cultured with Raji target cells for 5 hours at various effector-to-target (E:T) ratios and analyzed for expression of markers of activation and cytotoxicity. CAR-NK cells produced more inflammatory cytokines IFN $\gamma$ and TNFa than CAR-negative NK cells after co-culture (Figure 3A and 3B). CAR-NK cells also expressed more CD107a on their surface, a marker of 
degranulation (Figure 3C). In a luciferase-based killing assay, CAR-NK cells killed over $90 \%$ of Raji cells in 24 hours at the lowest E:T ratio of 1:3 (Figure 3D). These data show robust functionality of CAR-NK cells against CD19-expressing target cells.

Our ultimate goal is to deploy this manufacturing pipeline for clinical use, requiring cryopreservation and banking of manufactured CAR-NK cells. Recent studies have shown that while cryopreservation may have little effect on NK viability, it can cause a loss of cytotoxic function ${ }^{55}$. Thus, we tested cytotoxicity of CAR-NK cells immediately after thaw or after overnight culture in media containing $100 \mathrm{IU} / \mathrm{mL}$ IL2 (Figure 3D). Although cytotoxicity of CAR-NK cells immediately after thaw was reduced compared to fresh cells, target cell killing was still efficient at higher E:T ratios (3:1). Cytotoxicity of frozen CAR-NK cells was restored to that of fresh cells after an overnight rest in media containing IL2. We did not observe a reduction in cell number after the overnight rest, but we did observe a slight reduction in cell viability (Supplemental Figure 4A and 4B). These data provide further evidence for loss of NK function from cryopreservation, but suggest that allowing NK cells to recover after thaw or delivering higher doses of NK cells are approaches to circumvent this loss of function.

\section{Efficient Engineering of CAR-T Cells using Tc Buster Transposition}

A long-term goal of our work is to develop a combination therapy of CAR-NK and CAR-T cells. Thus, we next evaluated the Tc Buster transposon system for the manufacture of CAR-T cells. We stimulated CD3+ primary human $\mathrm{T}$ cells with 11 
$\alpha \mathrm{CD} 3 / \alpha \mathrm{CD} 28$ DynaBeads for 2 days and electroporated them with the nanoplasmid alone or in combination with $\mathrm{TcB}$ or TcB-M mRNA. Three days post-electroporation, we selected for transposon integration with $250 \mathrm{nM}$ MTX for an additional 7 days, for a total production timeline of 12 days (Figure 4A). We observed successful enrichment (>99\% $\mathrm{CAR}+$ ) of engineered cells with MTX while maintaining high cell viability (Figure 4B 4D). We similarly tested CAR-T cells in a killing assay against CD19-expressing Raji cells and observed near complete killing of target cells at higher E:T ratios (3:1) (Figure 4E).

\section{Discussion}

Current methods for the manufacture of CAR-NK and CAR-T cells for clinical use largely rely on viral transduction. The nature of NK cells as first responders to viral infection has likely led them to evolve resistance to viral infection and explains their intransigence to viral transduction ${ }^{\mathbf{5 6}}$. In addition, the use of viral vectors for CAR-NK and CAR-T production has several downsides. Production and handling of viral vectors is time consuming and costly, and viral vectors are limited in the size and complexity of their cargo and carry the risk of insertional mutagenesis. Engineering using transposons is an attractive alternative approach as production is convenient and cost-effective, and transposons have been shown to have a safer integration profile with reduced preference for integration near gene regulatory elements ${ }^{24}$. 
The goal of our work was to develop a protocol for manufacturing CAR-NK and CAR-T cells using the Tc Buster transposon system. The Tc Buster transposon is a member of the hAT superfamily of transposons and was originally isolated from the red flour beetle. Tc Buster has been optimized for transposition in mammalian cells and a hyperactive mutant (TcB-M) has been evolved by Bio-Techne (Minneapolis, MN). We obtained wild-type TcB and TcB-M mRNA from Bio-Techne and used them to deliver a model expression cassette containing a second generation CD19-CAR, mutant DHFR, and EGFP flanked by Tc Buster inverted terminal repeats (ITRs) to primary human PB NK and T cells.

The use of transposons in NK cells has been limited by DNA toxicity ${ }^{1}$. To avoid this, we delivered the transposase as modified $\mathrm{mRNA}^{57}$ and the transposon via nanoplasmid vectors which have a small backbone, high supercoiling, and are readily scaled under cGMP compliance ${ }^{58}$. We also optimized activation, electroporation, recovery, and expansion conditions to achieve $49.63 \%$ ( $\pm 4.64 \%)$ integration efficiency in NK cells without selection (Figure 1). One such modification we made to our protocol included pre-treating NK cells with an RNase inhibitor prior to electroporation. This enhanced transposition efficiency from $24.05 \%( \pm 1.36 \%)$ to $49.63 \%( \pm 4.64 \%)$. This is likely due to degradation of the transposase mRNA by RNases produced by NK cells without RNase inhibition. However, there is very limited data available on the expression of ribonucleases in NK cells in resting or activated states. Because of this, we used a 
broad RNase inhibitor which targeted RNase A, RNase B, and RNase T2 in our workflow $^{51}$. In future studies, it would be useful to profile NK cells for expression of a full panel of RNases at resting state and during several activation timepoints, as there may be other RNases produced by NK cells that could be targeted to further enhance efficiency.

We included a MTX-resistant DHFR mutein (L22F, F31S) in our cargo ${ }^{54}$. This allowed us to enrich our engineered population using MTX, without the need for GMP-compliant cell sorting facilities or clinical-grade monoclonal antibodies. Further, MTX is approved for clinical use and is being as described here for ex vivo enrichment of engineered cells (NCT04483778) (Figure 2). Overall, we developed a CAR-NK cell manufacturing protocol that includes 4 days of feeder cell activation before electroporation, re-expansion 2 days after electroporation, and a third expansion with MTX selection (Figure 2A). This process results in $\sim 1400$-fold expansion from electroporation input when cells are engineered with TcB-M (Figure 2D). Current clinical trials using CAR-NK cells have treated patients with doses ranging from $1 \times 10^{5}-1 \times 10^{7}$ cells/kg of body weight ${ }^{59}$. Thus, the average North American patient $(80 \mathrm{~kg}$ ) would receive a dose of $8 \times 10^{6} \mathrm{NK}$ cells on the low end and $8 \times 10^{8} \mathrm{NK}$ cells on the high end. A single dose of $1 \times 10^{7}$ cells $/ \mathrm{kg}$ could be produced in 20 days from a starting number of just $5.8 \times 10^{5} \mathrm{NK}$ cells. We routinely obtain apheresis products from healthy donors containing an average of $1 \times 10^{9}$ NK cells ( $n=12$ donors). Thus, we could generate an average of 1724 high $\left(1 \times 10^{7}\right.$ cells $\left./ \mathrm{kg}\right)$ doses from each donor. 14 
Importantly, we validated the function of CAR-NK cells produced using our protocol in standard in vitro assays. CAR-NK cells showed high levels of degranulation, inflammatory cytokine production, and target cell killing compared to CAR-negative controls (Figure 3A - 3D). Interestingly, it has been shown that cryopreservation may lead to reduced cytotoxicity of $\mathrm{NK}$ cells ${ }^{55}$. An off-the-shelf product requires cryopreservation and banking of engineered cells. We did observe reduced cytotoxicity of CAR-NK cells immediately after thaw, but this phenotype was rescued after an overnight rest in media containing $100 \mathrm{IU} / \mathrm{mL}$ IL2 (Figure 3E). However, it is likely not feasible to include this type of overnight rest period in a clinical protocol. We observed efficient target cell killing immediately after thaw when we used higher E:T ratios. Therefore, instead of implementing an overnight rest in a clinical protocol, it is likely more practical to simply deliver a higher dose of cells. This is feasible as the administration of CAR-NK cells clinically has not been associated with cytokine release syndrome, neurotoxicity, or graft-versus-host disease (GvHD), and the maximum tolerated dose has not been reached $^{59}$.

Finally, we deployed the Tc Buster system for the production of CAR-T cells. CAR-T cells have been generated for preclinical and clinical use with a number of transposon systems including $\mathrm{SB}$ and $\mathrm{PB}^{\mathbf{2 5 - 2 8 , 3 0 - 3 2 , 3 5 , 3 6 , 4 3}}$. Here, we demonstrated the feasibility and high efficiency of their production using Tc Buster and validated their subsequent effector function (Figure 4). 
Our work provides a platform for robust delivery of multicistronic, large cargo via transposition to primary human $\mathrm{PB}$ NK and $\mathrm{T}$ cells. Our results demonstrate that CAR-expressing NK and T cells can be enriched using MTX selection at clinically relevant doses, while maintaining high viability and function, and we can manufacture a large number of doses in a matter of three weeks. This approach represents a versatile, safe, and cost-effective option for the manufacture of CAR-NK and CAR-T cells compared to viral production methods. Importantly, further studies are required to test Tc Buster delivery of larger cargo that exceeds the carrying capacity of viral vectors. Future work will focus on the validation of CAR-NK and CAR-T cells in preclinical in vivo models. We will also explore the use of other CARs targeting solid tumors and combining CAR-NK and CAR-T cells in a single infusion.

\section{Materials and Methods}

\section{Vectors and Reagents}

Tc Buster (TcB) and Hyperactive Tc Buster (TcB-M) sequences were obtained from Bio-Techne (Minneapolis, MN). CD19-DHFR-EGFP flanked by Tc Buster ITRs was cloned into a Nanoplasmid backbone (Nature Technology, Lincoln, NE).

\section{Donor T and NK Cell Isolation}

Peripheral blood mononuclear cells (PBMCs) from de-identified healthy human donors were obtained by automated leukapheresis (Memorial Blood Centers, Minneapolis, MN). 
CD3+ T cells or CD56+CD3- NK cells were isolated from the PBMC population using the EasySep Human T Cell Isolation Kit or EasySep Human NK Cell Isolation Kit (STEMCELL Technologies, Cambridge, MA). T cells were frozen at 1-2 x $10^{7}$ cells $/ \mathrm{mL}$ and NK cells were frozen at $5 \times 10^{6}$ cells $/ \mathrm{mL}$ in CryoStor CS10 (STEMCELL Technologies, Cambridge, MA) and thawed into culture as needed. Samples were obtained after informed consent with approval from the University of Minnesota Institutional Review Board (IRB 1602E84302)

\section{T Cell Culture}

$\mathrm{T}$ cells were cultured in OpTimizer CTS T Cell Expansion SFM containing 5\% CTS Immune Cell SR (ThermoFisher, Waltham, MA), L-Glutamine, Penicillin/Streptomycin (Lonza, Basel, Switzerland), $10 \mathrm{mM}$ N-Acetyl-L-cysteine (Sigma-Aldrich, St. Louis, MO), 300 IU/mL IL-2, 5 ng/mL IL7, and 5 ng/mL IL-15 (PeproTech, Rocky Hill, NJ). T cells were activated with DynaBeads Human T-Activator CD3/CD28 (ThermoFisher, Waltham, MA) at a 2:1 bead:cell ratio for 48 hours prior to electroporation. Following electroporation, T cells were re-stimulated with DynaBeads and maintained at $\sim 1 \times 10^{6}$ cells/mL.

\section{NK Cell Culture}

NK cells were cultured in CTS AIM V SFM containing 5\% CTS Immune cell SR (ThermoFisher, Waltham, MA), Penicillin/Streptomycin, and IL-2 (100 IU/mL). NK 
cells were activated by co-culture with X-irradiated (100 Gray) feeder cells (K562 expressing membrane-bound IL21 and 41BB-L $\left.{ }^{60}\right)$ at indicated feeder:NK ratios (2:1 prior to electroporation, 5:1 48 hours after electroporation, or 1:1 for all subsequent expansions).

\section{T Cell Electroporation}

After 48 hours of stimulation, DynaBeads were magnetically removed, and T cells were washed once with PBS prior to resuspension in electroporation buffer. The 4D-Nucleofector (Lonza, Basel, Switzerland) and P3 kit was used with 1 x $10^{6} \mathrm{~T}$ cells per $20 \mu \mathrm{L}$ cuvette, $1 \mu \mathrm{g}$ transposase mRNA, $1 \mu \mathrm{g}$ transposon nanoplasmid, and the Nucleofector program FI-115. Transposon nanoplasmid alone was used as a control for all experiments. $\mathrm{T}$ cells were allowed to recover in antibiotic-free medium containing 1 $\mu \mathrm{g} / \mathrm{mL}$ DNase I solution (STEMCELL Technologies, Cambridge, MA) at $37^{\circ} \mathrm{C}, 5 \% \mathrm{CO} 2$ for 30 minutes following gene transfer, and then were cultured in complete $\mathrm{T}$ cell medium and re-stimulated with DynaBeads.

\section{Electroporation of activated NK cells}

Feeder cell-activated NK cells were washed once with PBS and resuspended at $3 \times 10^{7}$ cells/mL in electroporation buffer. Protector RNase inhibitor (Sigma Aldrich, St. Louis, MO) was added to the mixture at a concentration of $0.8 \mathrm{U} / \mu \mathrm{L}$ and incubated for 5 minutes at room temperature. The cell mixture was added to $1 \mu \mathrm{g}$ of transposase mRNA and $1 \mu \mathrm{g}$ 
transposon nanoplasmid on ice. Transposon nanoplasmid alone was used as a control for all experiments. This mixture was electroporated in a $10 \mu \mathrm{L}$ tip using the Neon Transfection System (ThermoFisher, Waltham, MA) under the following conditions: 1850 volts, pulse width of $10 \mathrm{~ms}$, two pulses. NK cells were allowed to recover at a density of $1.5 \times 10^{6}$ cells $/ \mathrm{mL}$ in antibiotic-free medium containing $1 \mathrm{ug} / \mathrm{mL}$ DNase I solution (STEMCELL Technologies, Cambridge, MA), and were then cultured in complete NK cell medium at a density of $6 \times 10^{5}$ cells $/ \mathrm{mL} .48$ hours after electroporation, NK cells were expanded with feeder cells at a 5:1 feeder:NK ratio.

\section{Antibodies and Flow Cytometry}

The following antibodies, proteins, and dyes were used: APC- or PE-conjugated anti-CD56 (clone REA196; Miltenyi Biotec), PE-conjugated anti-CD3 (clone SK7; BD Biosciences), Atto 647N-conjugated recombinant human CD19 (BioTechne), Brilliant violet 421-conjugated anti-IFN $\gamma$ (clone 4S.B3; BioLegend), APC-labeled anti-TNFa (clone Mab11, BioLegend), Brilliant violet 650-conjugated anti-CD107a (clone H4A3; BD Biosciences), SYTOX Blue dead cell stain (ThermoFisher), Fixable viability dye eFluor 780 (eBioscience). Flow cytometry assays were performed on a CytoFLEX S flow cytometer (Beckman Coulter) and all data were analyzed with FlowJo verson 10.4 software (FlowJo LLC).

NK Cell Functional Assays 
For intracellular cytokine staining, NK cells were plated at $2.5 \times 10^{6}$ cells $/ \mathrm{mL}$ in NK cell medium without cytokines. After incubation overnight, the CD19+ Burkitt's Lymphoma cell line Raji was added at the indicated effector-to-target (E:T) ratios. Brilliant violet Anti-CD107a was added to the culture and cells were incubated for 1 hour at $37{ }^{\circ} \mathrm{C}$. Brefeldin A and monensin (BD Biosciences, San Jose, CA) were added and cells were incubated for an additional 4 hours. Cells were stained with fixable viability dye, then for extracellular antigens. Cells were fixed and permeabilized using BD Cytofix/Cytoperm (BD Biosciences, San Jose, CA) following manufacturer's instructions. Cells were then stained for intracellular IFN $\gamma$ and $\mathrm{TNF} \alpha$ and analyzed by flow cytometry.

\section{Target Cell Killing Assays}

$\mathrm{T}$ cells or NK cells were cultured overnight in medium without cytokines. Luciferase-expressing Raji cells were seeded into a black round-bottom 96-well plate (3 x $10^{4}$ cells per well). T cells or NK cells were added to the wells in quadruplicate at the indicated E:T ratios. Target cells without effectors served as a negative control (spontaneous cell death) and target cells incubated with $1 \%$ NP-40 served as a positive control (maximum killing). Co-cultures were incubated at $37 \mathrm{C}$ for 24 hours. After incubation, D-luciferin (potassium salt; Gold Biotechnology, St. Louis, MO) was added to each well at a final concentration of $25 \mathrm{ug} / \mathrm{mL}$ and incubated for 10 minutes. Luminescence was read in endpoint mode using a BioTek Synergy microplate reader. 


\section{Statistical Analysis}

The Student's t-test was used to test for significant differences between two groups. Differences between 3 or more groups were tested by one-way ANOVA analysis followed by Tukey's post-hoc test. All assays were repeated in 3-5 independent donors. Mean values +/- standard error of the mean (SEM) are shown. The level of significance was set at $\alpha=0.05$. Statistical analyses were performed using GraphPad Prism 8.0.

\section{ACKNOWLEDGEMENTS}

We thank Bio-Techne for providing us with TcB-M and their support of these studies.

\section{AUTHOR CONTRIBUTIONS}

E.J.P, B.R.W, and B.S.M conceived of the project, designed experiments, and directed the research. E.J.P performed all experiments with assistance from W.S.L, B.J.W, N.J.S, and J.G.S. B.J.W and N.J.S performed T cell culture and electroporations. E.J.P performed all data analysis. E.J.P wrote the manuscript with input from all authors.

\section{CONFLICT OF INTEREST}

B.R.W and B.S.M are consultants for Bio-Techne. All other authors have no conflicts to declare. 

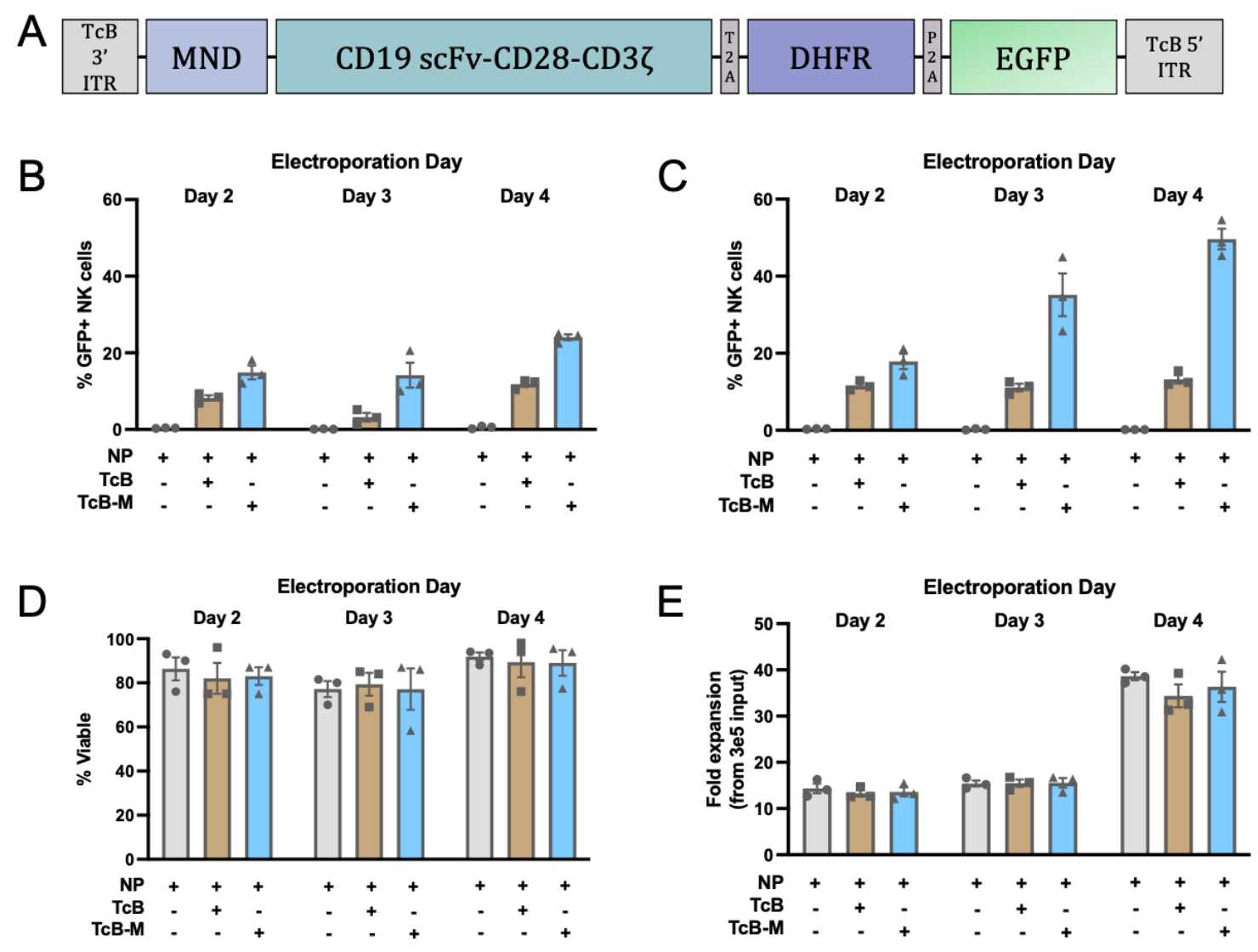

Figure 1. Delivery of a CD19-CAR-DHFR-EGFP transposon to NK cells using the Tc Buster Transposon System. (A) The $3.7 \mathrm{~kb}$ transposon flanked by Tc Buster ITRs, containing an MND promoter, second generation CD19 CAR, methotrexate resistant DHFR mutant, and enhanced GFP. Elements are separated by 2A ribosomal skip sequences. This cargo was cloned into a nanoplasmid (NP) backbone for delivery. (B-E) Primary human peripheral blood (PB) NK cells ( $\mathrm{n}=3$ human donors) were expanded for 2, 3, or 4 days with mbIL21- and 41BBL-expressing K562 feeder cells at a 2:1 (feeder:NK) ratio. NK cells were electroporated with the nanoplasmid transposon (NP) alone or in combination with mRNA encoding either Tc Buster (TcB) or the hyperactive mutant Tc Buster (TcB-M). Two days after electroporation, NK cells were expanded with feeder cells (5:1 feeder:NK ratio) for 1 week to allow for the loss of transient NP expression. After expansion, GFP expression was measured by flow cytometry, viability was measured by Trypan Blue exclusion, and fold expansion was calculated. (B) NK cells electroporated without pre-treatment with RNase inhibitor. (C-E) NK cells electroporated after 5-minute pre-treatment with RNase inhibitor. 
A

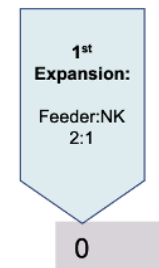

0

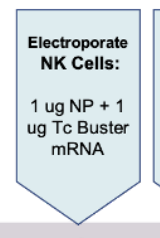

4

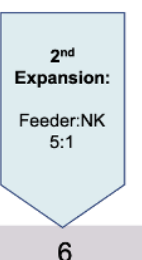

6

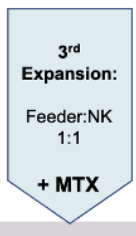

13

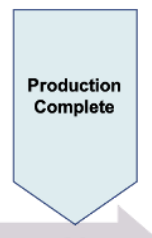

20

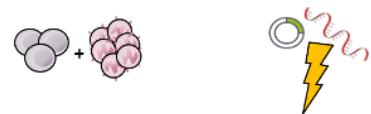<smiles>C1=C[Ge]C=C1</smiles>

$\infty$

B

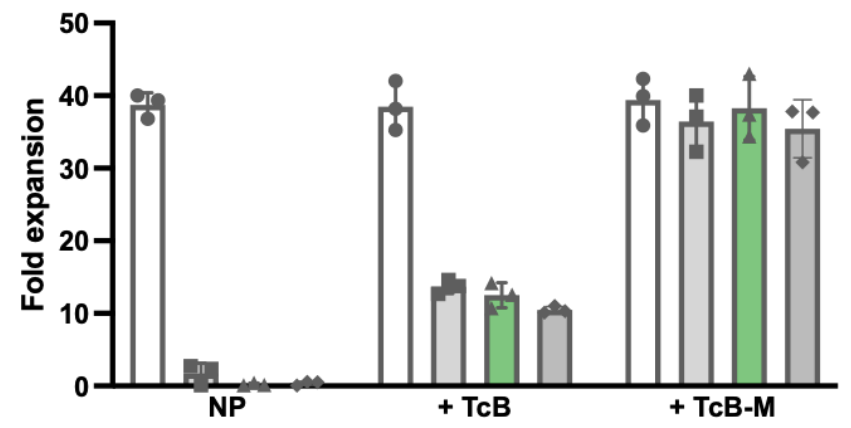

C

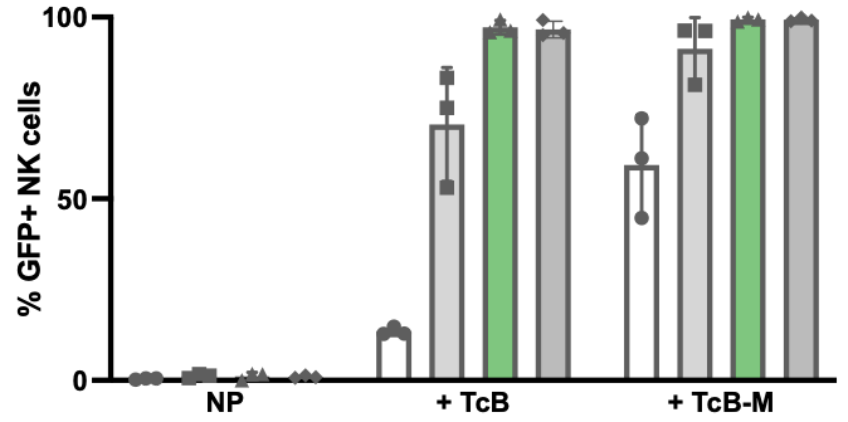

D

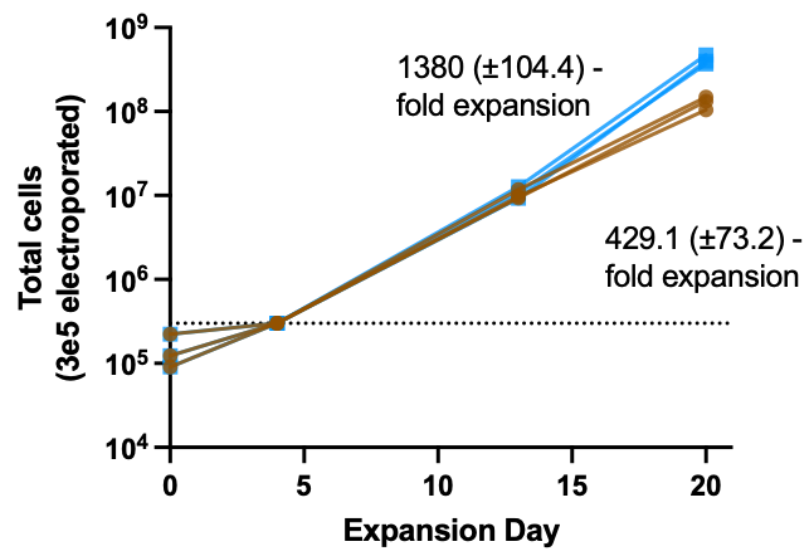

- No MTX

- $50 \mathrm{nM}$ MTX

- 250 nM MTX

- 500 nM MTX

- 50 nM MTX

- 250 nM MTX

- 500 nM MTX 
Figure 2. Efficient production of CAR-NK cells using an optimized engineering pipeline incorporating MTX selection. (A) Timeline for production of CAR-NK cells: Primary human peripheral blood NK cells ( $\mathrm{n}=3$ human donors) are activated with mbIL21- and 41BBL-expressing K562 feeder cells at a 2:1 (feeder:NK) ratio for 4 days. On day 4, NK cells are treated with RNase inhibitors and electroporated with transposition reagents. Two days after electroporation, NK cells are expanded for one week using a 5:1 (feeder:NK) ratio. NK cells are expanded again for one week at a 1:1 (feeder:NK) ratio in media containing MTX. (B) NK cells were expanded a third time in media containing increasing doses of MTX and fold expansion was calculated from input into the third expansion in order to determine optimal MTX selection dose. (C) GFP expression was measured by flow cytometry. (D) Total cells were tracked over the course of the full production timeline and fold expansion was calculated from electroporation input. 

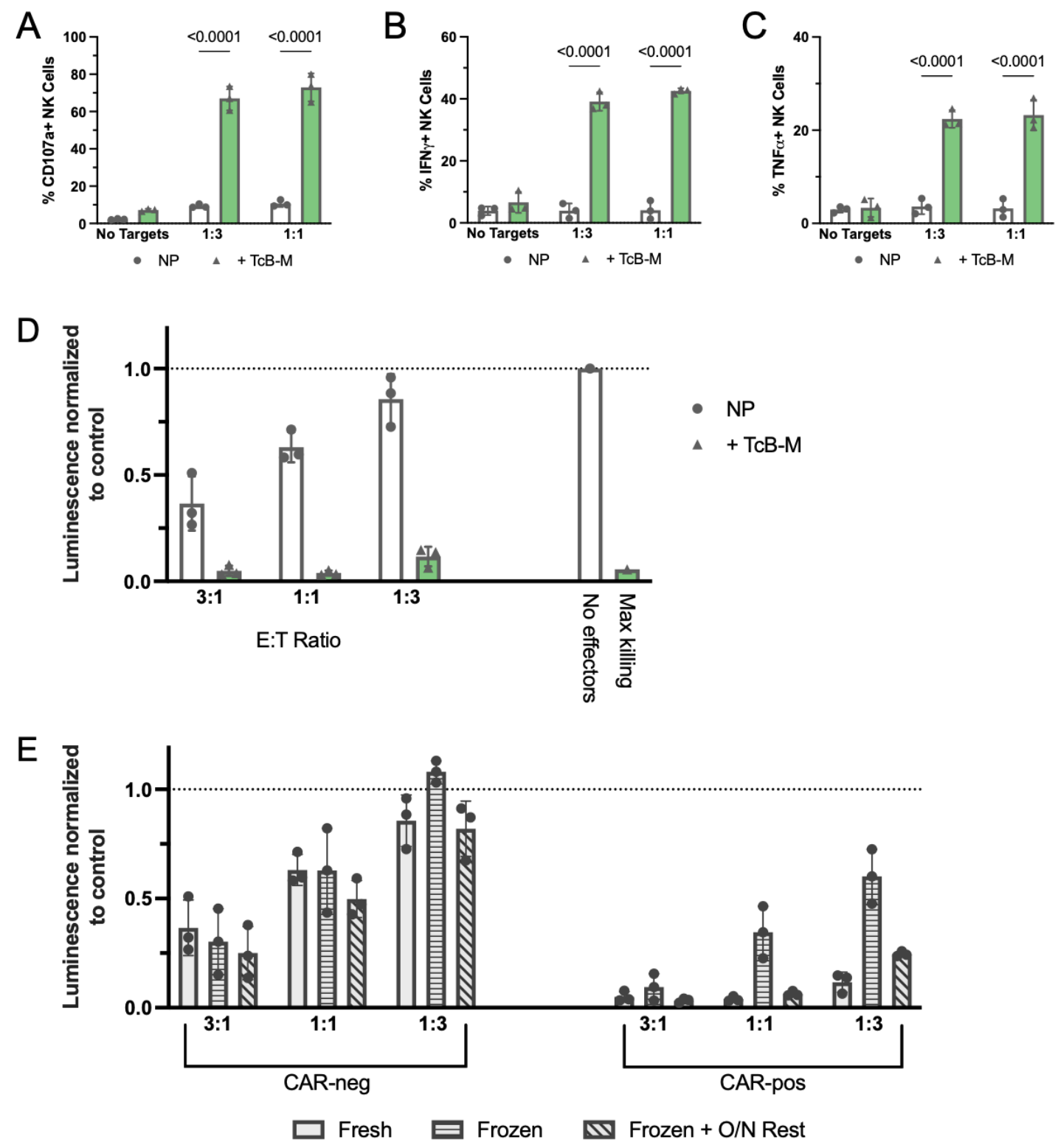

Figure 3. CD19-CAR-expressing NK cells show enhanced activity and tumor cell killing compared to CAR-negative controls. CAR-positive cells were engineered with TcB-M and selected with $250 \mathrm{nM}$ MTX. CAR-negative control cells were electroporated with NP alone and were not selected with MTX. (A-C) CAR-positive and CAR-negative NK cells were co-cultured with CD19+ Raji cells at the indicated effector-to-target (E:T) ratios for 5 hours. Co-cultures were set up in triplicate. After co-culture, NK cells were analyzed by intracellular flow cytometry for the expression of CD107a (A), IFN $\gamma$ (B), and $\mathrm{TNF} \alpha$ (C). (D-E) CAR-positive and CAR-negative NK cells were co-cultured with 
luciferase-expressing CD19+ Raji cells at the indicated E:T ratios for 24 hours. Co-cultures were set up in quadruplicate. After co-culture, cells were incubated with d-luciferin for 10 minutes and luminescence was read on a plate reader. The assay was performed with fresh NK cells immediately after production and with cryopreserved NK cells immediately after thaw or after an overnight rest in media containing $100 \mathrm{IU} / \mathrm{mL}$ IL2. 


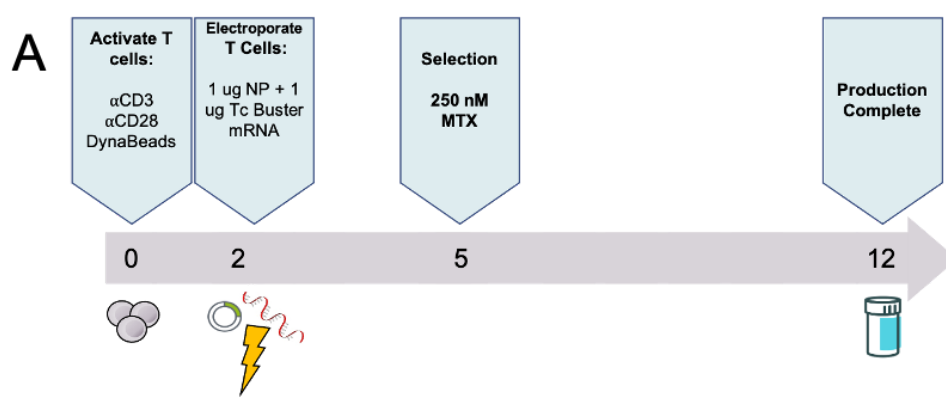

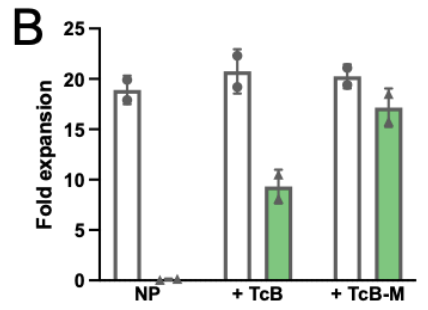

- No MTX $\triangle 250$ nM MTX

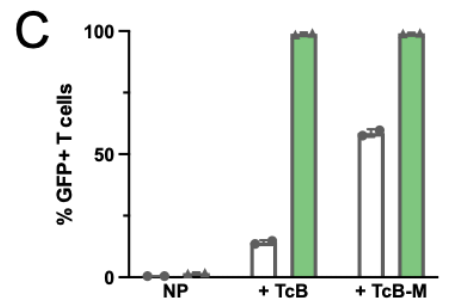

- NoMTX $\triangle 250 \mathrm{nM}$ MTX

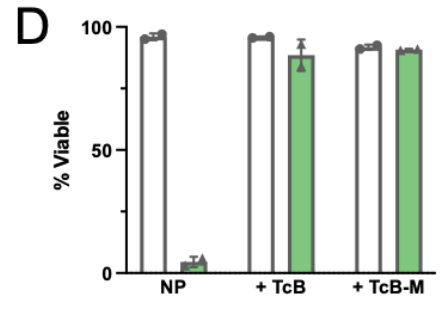

- No MTX $\triangle 250 \mathrm{nM}$ MTX

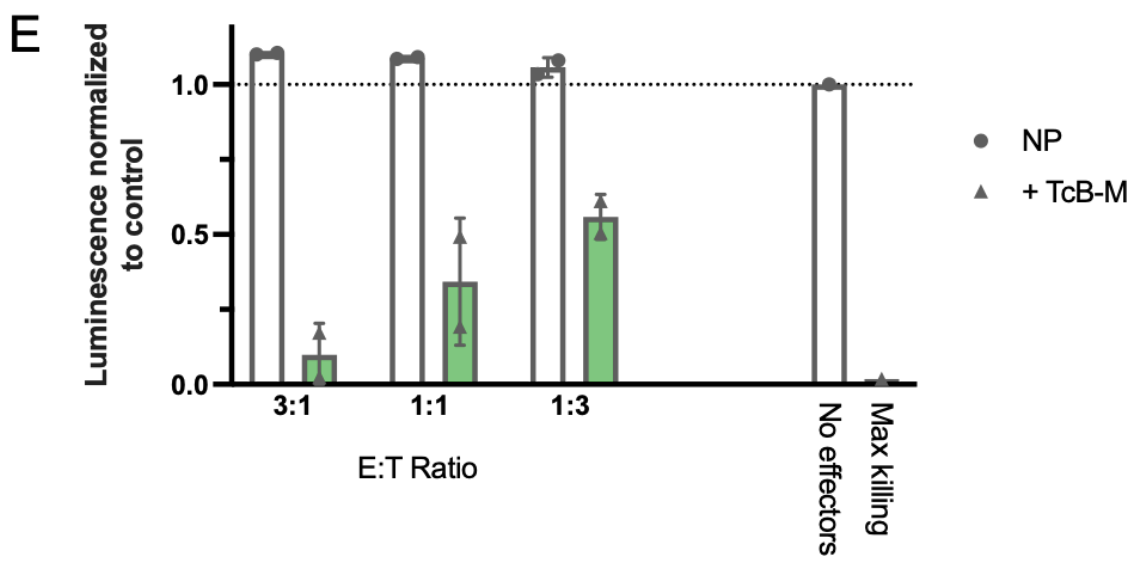

Figure 4. CD19-CAR $T$ cells can be generated using Tc Buster transposition and have functional killing against tumor targets. (A) Timeline for production of CAR-T cells: Primary human peripheral blood $\mathrm{T}$ cells are activated with $\alpha \mathrm{CD} 3 / \alpha \mathrm{CD} 28$ DynaBeads for 2 days. On day 2, DynaBeads are removed, and T cells are electroporated with transposition reagents and subsequently returned to DynaBead-containing cultures. 3 days after electroporation, MTX is added to the media at a concentration of $250 \mathrm{nM} .7$ days after MTX selection, production is complete and T cells are cryopreserved. (B-D) Production was performed with and without MTX selection. At the end of production, T cells were counted and fold expansion from electroporation input was calculated (B), GFP expression was measured by flow cytometry (C), and viability was measured by trypan blue exclusion (D). CAR-positive cells were engineered with TcB-M and selected with $250 \mathrm{nM}$ MTX. CAR-negative control cells were electroporated with NP alone and 
were not selected with MTX. CAR-positive and CAR-negative T cells were co-cultured with luciferase-expressing CD19+ Raji cells at the indicated E:T ratios for 24 hours. Co-cultures were set up in quadruplicate ( $\mathrm{n}=2$ donors). Co-cultures were incubated with D-luciferin for 10 minutes and luminescence was read on a plate reader. 


\section{Supplemental Material}
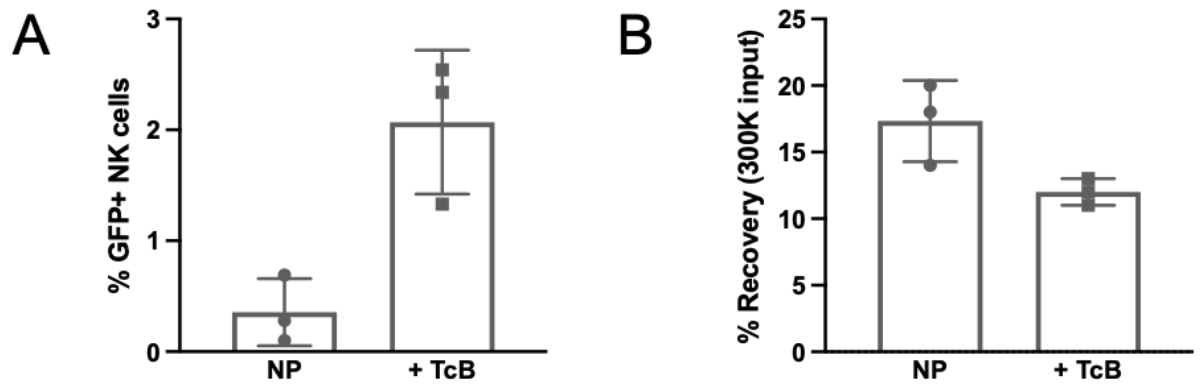

Supplemental Figure 1. Transposition efficiency and cell health are poor when cells are electroporated on day 7 of activation. Primary human peripheral blood (PB) NK cells were expanded for 7 days with mbIL21- and 41BBL-expressing K562 feeder cells at a 2:1 (feeder:NK) ratio. NK cells were electroporated with the nanoplasmid transposon (NP) alone or in combination with mRNA encoding Tc Buster (TcB). Two days after electroporation, NK cells were expanded with feeder cells (1:1 feeder:NK ratio) for 1 week to allow for the loss of transient NP expression. After this expansion, GFP expression was measured by flow cytometry (A) and cells were counted to calculate recovery from electroporation input (B).

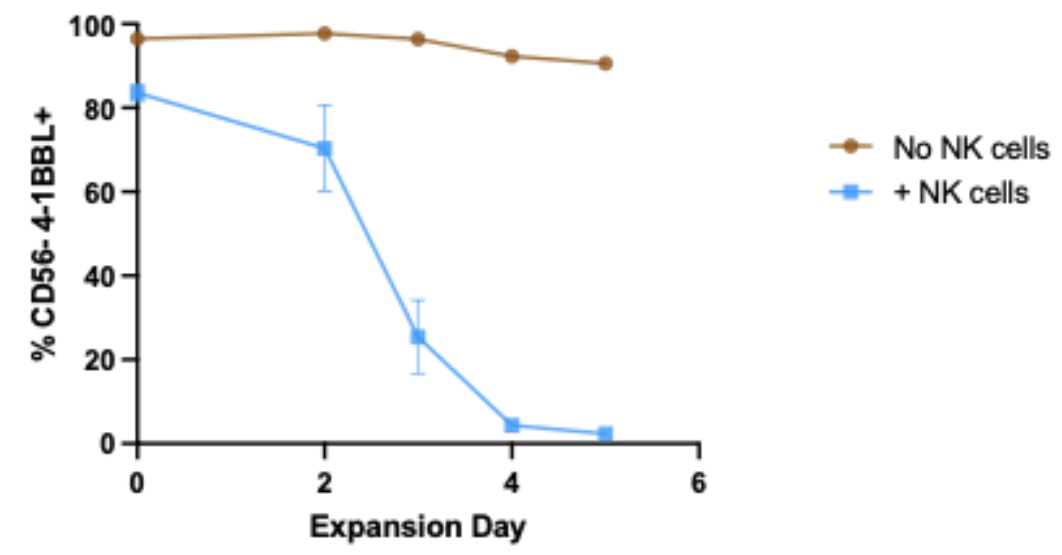

Supplemental Figure 2. Feeder cells are eliminated during co-culture with NK cells. K562 feeder cells expressing mbIL21 and 41BBL were irradiated (100 Gray) and 
cultured alone or in the presence of primary human peripheral blood NK cells (feeder-to-NK ratio $=5: 1$ ). Cultures were stained with antibodies for 41BB-L and NK cell marker CD56 and analyzed by flow cytometry. N=3 human NK cell donors.

Donor A

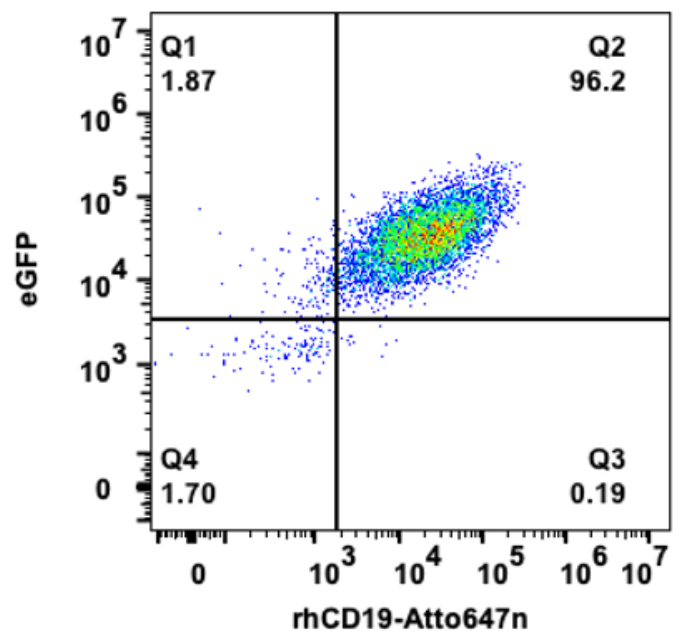

Donor B

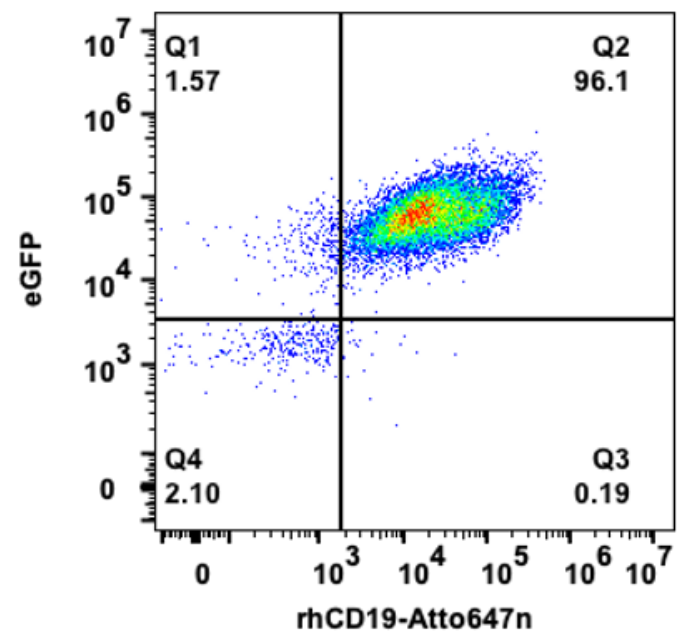

Supplemental Figure 3. Representative flow plots showing CD19-CAR staining using recombinant human CD19 (rhCD19) conjugated to fluorescent Atto 647N. CAR-NK cells were engineered usingTcB-M and selected with $250 \mathrm{nM}$ MTX. Cells were incubated with rhCD19 Atto 647N and analyzed by flow cytometry.
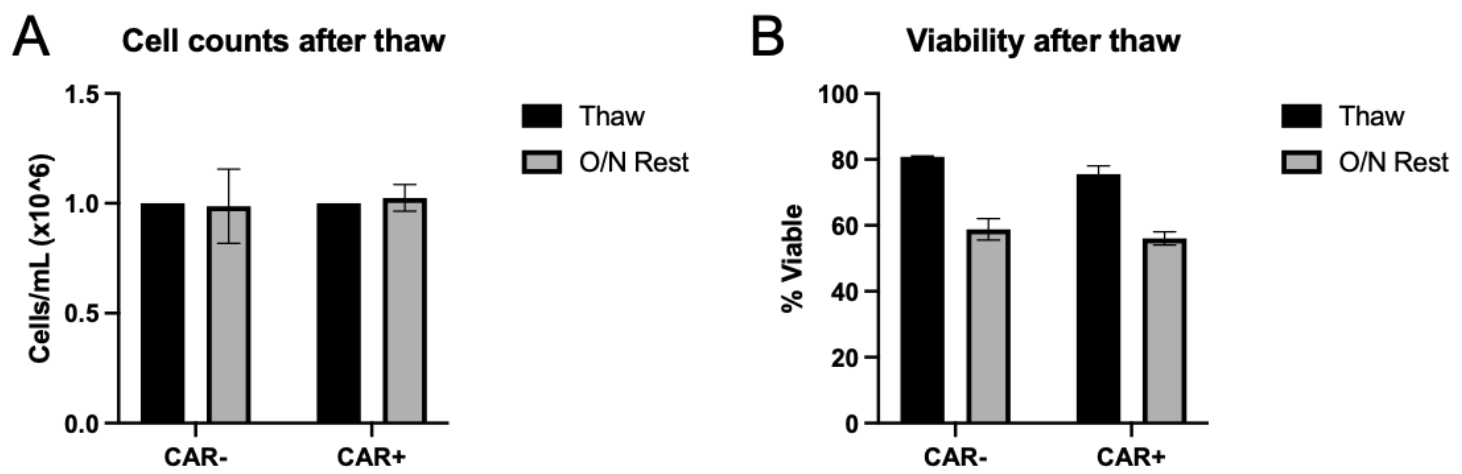
Supplemental Figure 4. NK cell viability and recovery after cryopreservation. CAR-positive and CAR-negative NK cells were cryopreserved after the 20-day production timeline. After thaw, cells were counted (A), and viability was measured by trypan blue exclusion (B). Cells were immediately plated in killing assays or plated at a density of $1 \times 10^{6}$ cells/mL in media containing $100 \mathrm{IU} / \mathrm{mL}$ IL2 overnight. After overnight rest, cells were counted, and viability was measured again. 


\section{References}

1. Robbins, G. M., Wang, M., Pomeroy, E. J. \& Moriarity, B. S. Nonviral genome engineering of natural killer cells. Stem Cell Res Ther 12, 350 (2021).

2. Ivics, Z., Hackett, P. B., Plasterk, R. H. \& Izsvák, Z. Molecular Reconstruction of Sleeping Beauty, a Tc1-like Transposon from Fish, and Its Transposition in Human Cells. Cell 91, 501-510 (1997).

3. Wilson, M. H., Coates, C. J. \& George, A. L. PiggyBac Transposon-mediated Gene Transfer in Human Cells. Molecular Therapy 15, 139-145 (2007).

4. Ni, J. et al. Active recombinant Tol2 transposase for gene transfer and gene discovery applications. Mob DNA 7, (2016).

5. Tsukahara, T. et al. The Tol2 transposon system mediates the genetic engineering of T-cells with CD19-specific chimeric antigen receptors for B-cell malignancies. Gene Therapy 22, 209-215 (2015).

6. Woodard, L. E. et al. Comparative analysis of the recently discovered hAT transposon TcBuster in human cells. PLoS One 7, e42666 (2012).

7. Arensburger, P. et al. Phylogenetic and functional characterization of the hAT transposon superfamily. Genetics 188, 45-57 (2011).

8. Li, X. et al. A resurrected mammalian hAT transposable element and a closely related insect element are highly active in human cell culture. Proc Natl Acad Sci U S A 110, E478-487 (2013).

9. Cartier, N. et al. Hematopoietic stem cell gene therapy with a lentiviral vector in X-linked adrenoleukodystrophy. Science 326, 818-823 (2009).

10. Cavazzana-Calvo, M. et al. Transfusion independence and HMGA2 activation after gene therapy of human $\beta$-thalassaemia. Nature 467, 318-322 (2010).

11. Biffi, A. et al. Lentiviral hematopoietic stem cell gene therapy benefits metachromatic leukodystrophy. Science 341, 1233158 (2013).

12. Sessa, M. et al. Lentiviral haemopoietic stem-cell gene therapy in early-onset metachromatic leukodystrophy: an ad-hoc analysis of a non-randomised, open-label, phase 1/2 trial. Lancet 388, 476-487 (2016).

13. Aiuti, A. et al. Lentiviral hematopoietic stem cell gene therapy in patients with Wiskott-Aldrich syndrome. Science 341, 1233151 (2013).

14. Bobisse, S. et al. Reprogramming $\mathrm{T}$ lymphocytes for melanoma adoptive immunotherapy by T-cell receptor gene transfer with lentiviral vectors. Cancer Res 69, 9385-9394 (2009).

15. Maude, S. L. et al. Chimeric antigen receptor $\mathrm{T}$ cells for sustained remissions in leukemia. N Engl J Med 371, 1507-1517 (2014). 
16. Turtle, C. J. et al. CD19 CAR-T cells of defined CD4+:CD8+ composition in adult B cell ALL patients. J Clin Invest 126, 2123-2138 (2016).

17. Kochenderfer, J. N. et al. Anti-CD19 CAR T Cells Administered after Low-Dose Chemotherapy Can Induce Remissions of Chemotherapy-Refractory Diffuse Large B-Cell Lymphoma. Blood 124, 550 (2014).

18. Staal, F. J. T., Pike-Overzet, K., Ng, Y. Y. \& van Dongen, J. J. M. Sola dosis facit venenum. Leukemia in gene therapy trials: a question of vectors, inserts and dosage? Leukemia 22, 1849-1852 (2008).

19. Staal, F. J. T., Aiuti, A. \& Cavazzana, M. Autologous Stem-Cell-Based Gene Therapy for Inherited Disorders: State of the Art and Perspectives. Front Pediatr 7, 443 (2019).

20. Hacein-Bey-Abina, S. et al. LMO2-Associated Clonal T Cell Proliferation in Two Patients after Gene Therapy for SCID-X1. Science 302, 415-419 (2003).

21. Gaspar, H. B. et al. Gene therapy of X-linked severe combined immunodeficiency by use of a pseudotyped gammaretroviral vector. The Lancet 364, 2181-2187 (2004).

22. Fraietta, J. A. et al. Disruption of TET2 Promotes the Therapeutic Efficacy of CD19-targeted T-cells. Nature 558, 307 (2018).

23. Hacein-Bey-Abina, S. et al. Insertional oncogenesis in 4 patients after retrovirus-mediated gene therapy of SCID-X1. J Clin Invest 118, 3132-3142 (2008).

24. Gogol-Döring, A. et al. Genome-wide Profiling Reveals Remarkable Parallels Between Insertion Site Selection Properties of the MLV Retrovirus and the piggyBac Transposon in Primary Human CD4(+) T Cells. Mol Ther 24, 592-606 (2016).

25. Singh, H. et al. Redirecting Specificity of T-Cell Populations For CD19 Using the Sleeping Beauty System. Cancer Res 68, 2961-2971 (2008).

26. Jin, Z. et al. The hyperactive Sleeping Beauty transposase SB100X improves the genetic modification of T cells to express a chimeric antigen receptor. Gene Therapy 18, 849-856 (2011).

27. Kebriaei, P. et al. Phase I trials using Sleeping Beauty to generate CD19-specific CAR T cells. J Clin Invest 126, 3363-3376 (2016).

28. Srour, S. A. et al. Long-term outcomes of Sleeping Beauty-generated CD19-specific CAR T-cell therapy for relapsed-refractory B-cell lymphomas. Blood 135, 862-865 (2020).

29. Crossland, D. L. et al. Antitumor activity of CD56-chimeric antigen receptor T cells in neuroblastoma and SCLC models. Oncogene 37, 3686 (2018).

30. Chicaybam, L. et al. CAR T Cells Generated Using Sleeping Beauty Transposon Vectors and Expanded with an EBV-Transformed Lymphoblastoid Cell Line Display Antitumor Activity In Vitro and In Vivo. Human Gene Therapy 30, 511-522 (2019).

31. Wang, P. et al. PiggyBac-engineered T cells expressing a glypican-3-specific chimeric antigen receptor show potent activities against hepatocellular carcinoma. Immunobiology 225, 151850 (2020). 
32. Bishop, D. C. et al. PiggyBac-Engineered T Cells Expressing CD19-Specific CARs that Lack IgG1 Fc Spacers Have Potent Activity against B-ALL Xenografts. Molecular Therapy 26, 1883-1895 (2018).

33. He, J. et al. Engineered CAR T cells targeting mesothelin by piggyBac transposon system for the treatment of pancreatic cancer. Cellular Immunology 329, 31-40 (2018).

34. Hurton, L. V. et al. Tethered IL-15 augments antitumor activity and promotes a stem-cell memory subset in tumor-specific T cells. PNAS 113, E7788-E7797 (2016).

35. Clauss, J. et al. Efficient Non-Viral T-Cell Engineering by Sleeping Beauty Minicircles Diminishing DNA Toxicity and miRNAs Silencing the Endogenous T-Cell Receptors. Human Gene Therapy 29, 569-584 (2018).

36. Nakazawa, Y. et al. Optimization of the PiggyBac Transposon System for the Sustained Genetic Modification of Human T-Lymphocytes. J Immunother 32, 826-836 (2009).

37. Darquet, A.-M., Cameron, B., Wils, P., Scherman, D. \& Crouzet, J. A new DNA vehicle for nonviral gene delivery: supercoiled minicircle. Gene Therapy 4, 1341 (1997).

38. Hudecek, M. et al. Minicircle-Based Engineering of Chimeric Antigen Receptor (CAR) T Cells. Recent Results Cancer Res. 209, 37-50 (2016).

39. Cheng, C. et al. Bacteria-free minicircle DNA system to generate integration-free CAR-T cells. Journal of Medical Genetics 56, 10-17 (2019).

40. Rakowski, S. A. \& Filutowicz, M. Plasmid R6K Replication Control. Plasmid 69, 231-242 (2013).

41. Luke, J., Carnes, A. E., Hodgson, C. P. \& Williams, J. A. Improved antibiotic-free DNA vaccine vectors utilizing a novel RNA based plasmid selection system. Vaccine 27, 6454-6459 (2009).

42. Williams, J. A., Luke, J., Johnson, L. \& Hodgson, C. pDNAVACCultra vector family: high throughput intracellular targeting DNA vaccine plasmids. Vaccine $\mathbf{2 4}$, 4671-4676 (2006).

43. Wang, J., Lupo, K. B., Chambers, A. M. \& Matosevic, S. Purinergic targeting enhances immunotherapy of CD73 + solid tumors with piggyBac-engineered chimeric antigen receptor natural killer cells. Journal for ImmunoTherapy of Cancer 6, 136 (2018).

44. Rotiroti, M. C. et al. Targeting CD33 in Chemoresistant AML Patient-Derived Xenografts by CAR-CIK Cells Modified with an Improved SB Transposon System. Mol Ther 28, 1974-1986 (2020).

45. Innate or Adaptive Immunity? The Example of Natural Killer Cells I Science. https://science-sciencemag-org.ezp1.lib.umn.edu/content/331/6013/44.long.

46. Matosevic, S. Viral and Nonviral Engineering of Natural Killer Cells as Emerging Adoptive Cancer Immunotherapies. J Immunol Res 2018, 4054815 (2018). 
47. Imamura, M. et al. Autonomous growth and increased cytotoxicity of natural killer cells expressing membrane-bound interleukin-15. Blood 124, 1081-1088 (2014).

48. Blocking NK Cell Inhibitory Self-Recognition Promotes Antibody-Dependent Cellular Cytotoxicity in a Model of Anti-Lymphoma Therapy I The Journal of Immunology. https://www.jimmunol.org/content/180/9/6392.long.

49. Pomeroy, E. J. et al. A Genetically Engineered Primary Human Natural Killer Cell Platform for Cancer Immunotherapy. Molecular Therapy (2019) doi:10.1016/j.ymthe.2019.10.009.

50. Marcus, A. et al. Tumor-derived cGAMP triggers a STING-mediated interferon response in non-tumor cells to activate the NK cell response. Immunity 49, 754-763.e4 (2018).

51. Ackermann, M. R. Chapter 3 - Inflammation and Healing1. in Pathologic Basis of Veterinary Disease (Sixth Edition) (ed. Zachary, J. F.) 73-131.e2 (Mosby, 2017). doi:10.1016/B978-0-323-35775-3.00003-5.

52. Ng, S. K. Generation of High-Expressing Cells by Methotrexate Amplification of Destabilized Dihydrofolate Reductase Selection Marker. in Protein Expression in Mammalian Cells: Methods and Protocols (ed. Hartley, J. L.) 161-172 (Humana Press, 2012). doi:10.1007/978-1-61779-352-3_11.

53. Affleck, J. G., Nowickyj, S. M. \& Walker, V. K. Selection for methotrexate resistance in mammalian cells bearing a Drosophila dihydrofolate reductase transgene: Methotrexate resistance in transgenic mammalian cells. Cell Biol Toxicol 26, 117-126 (2010).

54. Jonnalagadda, M. et al. Efficient selection of genetically modified human T cells using methotrexate-resistant human dihydrofolate reductase. Gene Therapy 20, 853-860 (2013).

55. Mark, C. et al. Cryopreservation impairs 3-D migration and cytotoxicity of natural killer cells. Nat Commun 11, 5224 (2020).

56. Lanier, L. L. Evolutionary struggles between NK cells and viruses. Nat Rev Immunol 8, 259-268 (2008).

57. Kocmik, I. et al. Modified ARCA analogs providing enhanced translational properties of capped mRNAs. Cell Cycle 17, 1624-1636 (2018).

58. Suschak, J. J. et al. Nanoplasmid Vectors Co-expressing Innate Immune Agonists Enhance DNA Vaccines for Venezuelan Equine Encephalitis Virus and Ebola Virus. Mol Ther Methods Clin Dev 17, 810-821 (2020).

59. Liu, E. et al. Use of CAR-Transduced Natural Killer Cells in CD19-Positive Lymphoid Tumors. New England Journal of Medicine 382, 545-553 (2020).

60. Denman, C. J. et al. Membrane-Bound IL-21 Promotes Sustained Ex Vivo Proliferation of Human Natural Killer Cells. PLOS ONE 7, e30264 (2012). 